\title{
Photon identification with the ATLAS detector
}

\author{
Gregor Geßner on behalf of the ATLAS collaboration \\ Technische Universität Dortmund \\ Otto-Hahn Straße 4a, 44227 Dortmund, Germany \\ E-mail: gregor.gessner@cern. ch
}

Good photon identification capabilities are important for many aspects of the ATLAS physics program at the LHC, from Higgs boson measurements to new physics searches. The identification of prompt photons and the rejection of background coming mostly from photons in hadron decays relies on the high granularity of the ATLAS calorimeter. Several data-driven methods are used to measure the efficiency of the photon identification requirements, covering a broad energy spectrum. At low energies, photons from radiative $Z$ boson decays are used. In the medium energy range, similarities between electron and photon showers are exploited using $Z \rightarrow e e$ decays. At high energies, inclusive photon samples are used. The results of these measurements performed in proton-proton collisions data at a center-of-mass energy of $\sqrt{s}=13 \mathrm{TeV}$ taken in 2015 and 2016 corresponding to an integrated luminosity of $\mathscr{L}=36.1 \mathrm{fb}^{-1}$ in the case of the radiative $Z$ boson and electron extrapolation method and $\mathscr{L}=37.1 \mathrm{fb}^{-1}$ for the matrix method are presented.

EPS-HEP 2017, European Physical Society conference on High Energy Physics 5-12 July 2017

Venice, Italy 


\section{Introduction}

Photons are important ingredients in many physics analyses at the ATLAS experiment [1] at the LHC. They participate in various Standard Model processes like $H \rightarrow \gamma \gamma$ and are used to search for hints of Beyond Standard Model physics. Photons are reconstructed from energy deposits in the calorimeter systems and information from the inner detector. It is differentiated between unconverted and converted photons, i.e. photons that have interacted with detector material upstream of the calorimeters and produced an electron-positron pair. Reconstructed photons are categorized as prompt or fake. Prompt photons originate from the hard scattering process or from the fragmentation of a parton. In contrast, any reconstructed photon arising from another source is considered as fake, e.g. the mis-reconstruction of a jet or an electron as a photon or photons from a decay of a hadron.

\section{Identification of photons}

Photon identification (ID) [2] is used to separate prompt from fake photons. The ID is based on a set of rectangular cuts on shower shape variables. These variables describe the lateral and longitudinal shower shape development in the electromagnetic calorimeter and the shower leakage fraction in the hadronic calorimeter. In simulations, shower shape variables are successfully, but imperfectly reproduced. The central values are shifted compared to data. To correct the simulation to data, factors are derived that describe back-shifts of the simulated distributions by sliding it to the distribution in data. To harmonize the photon ID, a common reference point is computed targeting the following criteria:

- high photon efficiency of about $85 \%$ for prompt photons with $\mathrm{E}_{T}>40 \mathrm{GeV}$;

- high multi-jet background rejection of about 5000;

- robustness against pile-up.

The Toolkit for Multivariate Data Analysis [3] is used to optimize the background rejection for a given signal efficiency. To ensure pile-up robustness, looser criteria are set on pile-up dependent variables, while tighter criteria are defined for pile-up independent variables.

\section{Photon identification efficiency measurements and scale factors}

The photon ID efficiency is defined as the fraction of prompt photons passing the tight ID selection. A scale factor is computed in order to correct the efficiency found in simulation to the efficiency determined in data. Three different data-driven methods are used to measure the efficiency in dedicated pseudorapidity and transverse energy intervals, separately for unconverted and converted photons:

Radiative $Z$ boson method: For this method, events with leptonic radiative $Z$ boson decays are selected covering a transverse energy range from 10 to $100 \mathrm{GeV}$. The selection provides a very pure photon sample. The number of signal and background events is determined with a 
template fit to the invariant mass distribution $m_{e e \gamma}$ or $m_{\mu \mu \gamma}$, respectively. The ID efficiency is determined in both channels and combined at the end.

Electron extrapolation method: This method covers a transverse energy range from 25 to 150 $\mathrm{GeV}$. Events with $Z$ bosons decaying into electrons are selected. The electron shower shapes are then mapped to photon shower shapes by the use of a Smirnov transform. The transformation parameters are obtained from simulation. The ID efficiency is measured with these photon shower shape variables. The strength of this method is the small uncertainty for converted photons since their shower shapes are very similar to the electron shower shapes.

Matrix method: The largest transverse energy range is covered by the matrix method from 25 up to $1500 \mathrm{GeV}$. Single photons are selected mainly stemming from quark-antiquark annihilation or Compton scattering. The ID efficiencies are expressed by efficiencies for prompt and fake photons for the track isolation that is weakly correlated with criteria on ID variables in the first layer of the electromagnetic calorimeter. The efficiency at high transverse energies comes along with small uncertainties.

The efficiencies derived by each method are compatible with each other within their uncertainty as shown in Figure 1 for unconverted and converted photons in the pseudorapidity interval of $|\eta|<0.6$ as an example. At higher energies prompt photons are easier to distinguish from fake photons, and the efficiency curve shows an increasing trend. Each method provides a set of scale factors that are combined with the BLUE technique [5]. It takes into account the uncertainties of each method as weights, while all uncertainties are treated as uncorrelated. The combined scale factors for the pseudorapidity region of $|\eta|<0.6$ are shown in Figure 2 for unconverted and converted photons, respectively. The dominant contribution at low transverse energies comes from the radiative $Z$ boson method. In the medium transverse energy range, the electron extrapolation method plays a major role. At high transverse energies, the matrix method takes over.
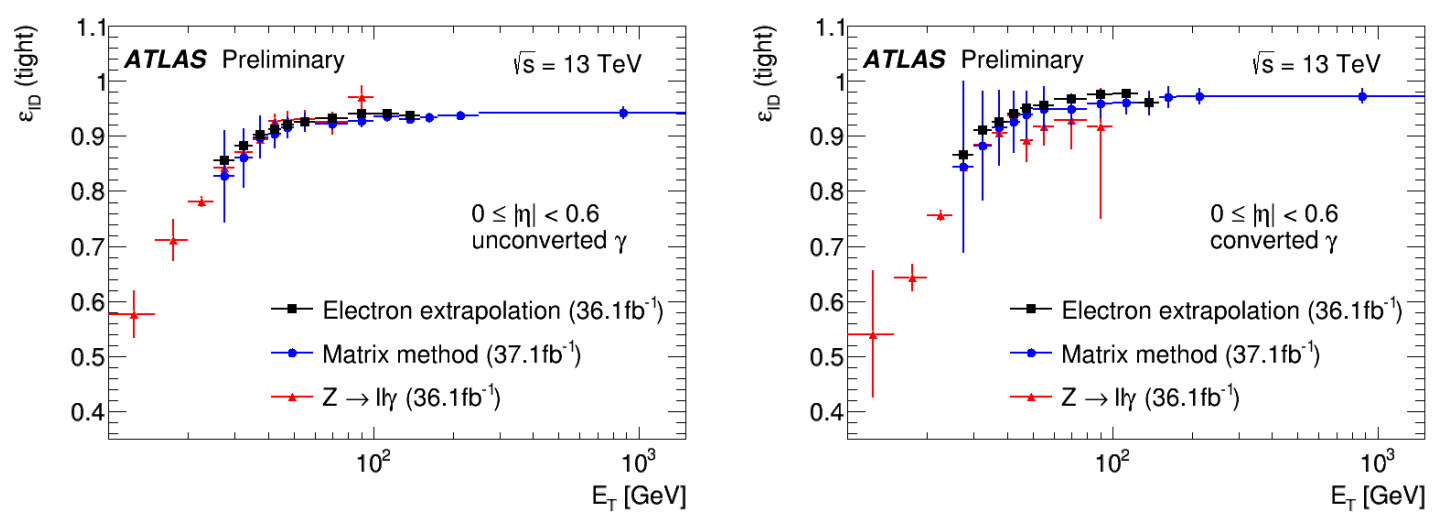

Figure 1: Comparison of the data-driven measurements (radiative $Z$ boson decay, electron extrapolation and matrix method) of the identification efficiency for unconverted (left) and converted photons (right) as a function of $E_{T}$ for the pseudorapidity interval $|\eta|<0$.6. For the matrix method, additional data were used when the toroid magnet was turned off that is not used for photon reconstruction. The error bars represent the sum in quadrature of the statistical and systematic uncertainties estimated with each method [4]. 

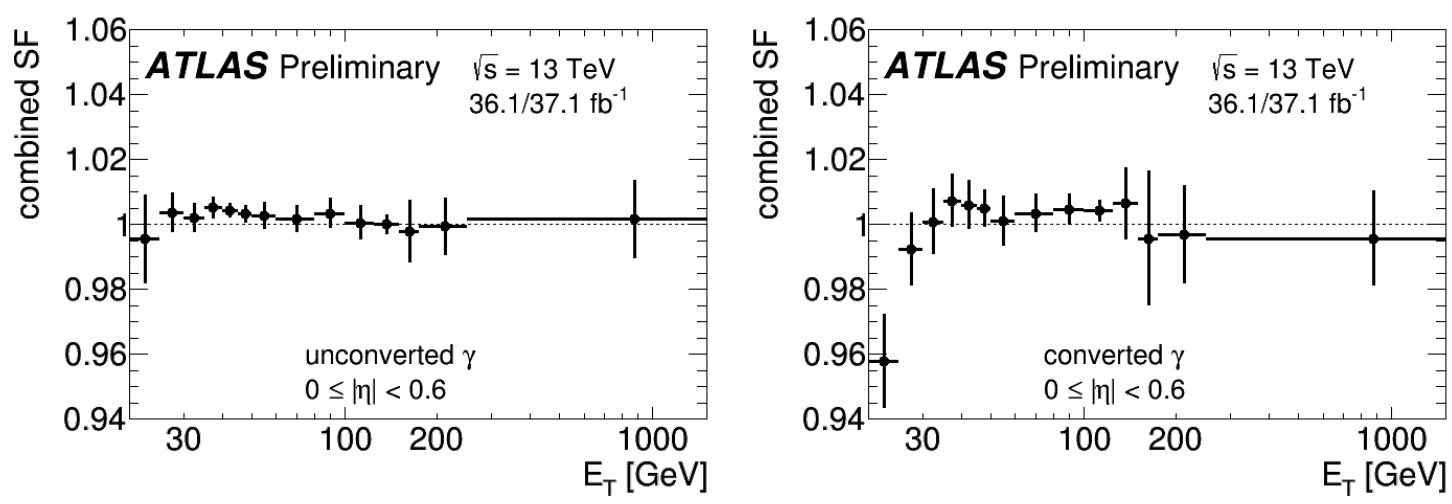

Figure 2: Data-to-MC identification efficiency ratio (SF) from the combination of the radiative $Z$ boson decay $\left(36.1 \mathrm{fb}^{1}\right)$, electron extrapolation $\left(36.1 \mathrm{fb}^{1}\right)$ and matrix method $\left(37.1 \mathrm{fb}^{1}\right)$ for unconverted (left) and converted photons (right) in the pseudorapidity interval $|\eta|<0.6$. For the matrix method, additional data were used when the toroid magnet was turned off that is not used for photon reconstruction. The uncertainty bars represent the sum in quadrature of the statistical and systematic uncertainties [4].

\section{Conclusion}

The photon identification strategy at ATLAS has been presented for data taken in 2015 and 2016 at $\sqrt{s}=13 \mathrm{TeV}$ corresponding to $\mathscr{L}=36.1 \mathrm{fb}^{-1}$ in the case of the radiative $Z$ boson and electron extrapolation and to $\mathscr{L}=37.1 \mathrm{fb}^{-1}$ for the matrix method. For the ID, criteria have been established on shower shape variables in the electromagnetic calorimeter. The photon ID efficiency has been measured with the three data-driven techniques radiative $Z$ boson decay, electron extrapolation and matrix method yielding compatible results. A combined set of scale factors has been obtained with the BLUE method taking into account the statistical and systematic uncertainties.

\section{Acknowledgment}

The author acknowledges the financial support by the Federal Ministry of Education and Research of Germany in the framework of ATLAS (FSP 103).

\section{References}

[1] ATLAS collaboration, The ATLAS Experiment at the CERN Large Hadron Collider, JINST 3 (2008) S08003.

[2] ATLAS collaboration, Photon identification in 2015 ATLAS data, ATL-PHYS-PUB-2016-015, https://cds.cern.ch/record/2203125

[3] A. Höcker et al., TMVA - Toolkit for Multivariate Data Analysis, PoS ACAT 040 (2007), arXiv:physics/0703039.

[4] ATLAS collaboration, Electron/Gamma Public Results, Webpage visited on July 20, 2017: https://twiki.cern.ch/twiki/bin/view/AtlasPublic/ElectronGammaPublicCollisionResults.

[5] L. Lyons, D. Gibaut, P. Clifford, How to combine correlated estimates of a single physical quantity, Nucl. Instr. and Meth. A 270 (1988) 110. 